\title{
Hunting for Dark Particles with Gravitational Waves
}

\author{
Gian F. Giudice ${ }^{1, a}$ \\ ${ }^{1}$ CERN, Theoretical Physics Department, Geneva, Switzerland
}

\begin{abstract}
The LIGO observation of gravitational waves from a binary black hole merger has begun a new era in fundamental physics. If new dark sector particles, be they bosons or fermions, can coalesce into exotic compact objects (ECOs) of astronomical size, then the first evidence for such objects, and their underlying microphysical description, may arise in gravitational wave observations. In this work we study how the macroscopic properties of ECOs are related to their microscopic properties, such as dark particle mass and couplings. We then demonstrate the smoking gun exotic signatures that would provide observational evidence for ECOs, and hence new particles, in terrestrial gravitational wave observatories. Finally, we discuss how gravitational waves can test a core concept in general relativity: Hawking's area theorem.
\end{abstract}

\section{Introduction}

The LIGO detection of GW150914 [1] - the first observed event of gravitational wave (GW) emission from binary black-hole merging - has ushered in a new era in the exploration of our universe. Not only does this result open new prospects for the study of the astrophysical properties of objects in extreme density conditions, but also allows for new ways of testing fundamental physics. Indeed, soon after the LIGO announcement, it was suggested that GW observations can be used to probe violations of the equivalence principle and Shapiro delay [2-5], modifications of gravity [4, 6-8], the presence of event horizons [9], the quantum structure of black holes [10], the hypothesis that dark matter is in the form of primordial black holes [11-13], the propagation of gravity waves [4, 14-21], phase transitions in the early universe $[22,23]$, neutrino properties from associated neutrino emission $[24,25]$, and axion clouds around black holes [26].

Here we want to investigate another way in which GW detection can contribute to fundamental science: the search for new physics in the form of exotic compact objects (ECO). Dark matter is a primary motivation to expect that new forms of stable matter must exist and be abundant in our universe. Moreover, quite independently of dark matter considerations, many new-physics theories predict the existence of stable or long-lived light particles with feeble interactions with ordinary matter. Because of their elusive nature, here we will generally refer to them as dark particles. It is conceivable that dark particles could coalesce into macroscopic objects. Although at present we are not able to predict the mass distribution of such exotic objects, it is possible that they could reach astronomical sizes. In this case GW detection from the merging of two ECOs or the collapse of an ECO into a stellar black hole could be used as a new and formidable way to probe the existence of dark particles and test their

a e-mail: Gian.Giudice@cern.ch 
properties. Actually, in some cases, this could be the only way in which dark particles are discovered, because their extremely feeble interactions make them completely invisible to collider searches or other particle physics experiments.

This presentation is based on ref. [27], where an extended discussion and a complete list of references can be found.

\section{Signatures of Exotic Compact Objects}

\subsection{LIGO Sensitivity on ECO parameters}

Let us consider two point masses $M_{1}$ and $M_{2}$ orbiting each other under the force of gravity. This is equivalent to a single body with reduced mass $\mu \equiv M_{1} M_{2} / M_{\text {tot }}$ (where $M_{\text {tot }}=M_{1}+M_{2}$ ) moving in an external gravitational potential. The equivalent body moves in an elliptic orbit with major semi-axis $l$, describing the separation of the two bodies.

The orbital period $P$ is related to $M_{\text {tot }}$ and $l$ by Kepler's third law

$$
P^{2}=\frac{4 \pi^{2} l^{3}}{M_{\mathrm{tot}}} .
$$

The frequency $f$ of GW emission is twice the orbital frequency $v=1 / P$, and thus is given by

$$
f=\sqrt{\frac{M_{\mathrm{tot}}}{\pi^{2} l^{3}}} .
$$

For the system of two BHs the innermost stable circular orbit (ISCO) is defined by

$$
R_{B H}^{\mathrm{ISCO}} \equiv 6 M_{\mathrm{tot}}
$$

The ISCO determines the end of the inspiral phase and the beginning of the merger phase, thus this radius characterises the typical frequency $f_{B H}^{\mathrm{ISCO}}$ expected in a binary merger. For a BH-BH merger, we find

$$
f_{B H}^{\mathrm{ISCO}}=\frac{1}{6^{3 / 2} \pi M_{\mathrm{tot}}} \quad(\text { for } \mathrm{BH}) .
$$

The maximum frequency at the end of inspiral, obtained from numerical simulations of the waveform of $\mathrm{BH}$ mergers, is given by $f=f_{B H}^{\mathrm{ISCO}}(1+\Delta)$, where the correction term $\Delta$ is computed in postNewtonian approximation and is a function of the mass ratio $M_{1} / M_{2}$ and of a single combination of the two BH spins. Its analytical expression can be found in [28]. The correction term $\Delta$ vanishes in the limit of negligible $\mathrm{BH}$ spin and large mass ratio. For non-rotating $\mathrm{BHs}$, it reaches a maximum value $\Delta=1.1$ for $M_{1} / M_{2}=3.56$, while $\Delta=0.7$ for equal BH masses. For spinning BHs, in the parameter range for which the expansion given in [28] is valid, the maximum value reached is $\Delta=3.1$. Thus, for discussion purposes, it is adequate to take the expression in eq. (4) as the frequency determining the end of inspiral. The correction term $\Delta$ is fully included in our numerical analysis.

By analogy with eq. (3), for ECOs we may define a typical GW frequency determined by the ISCO radius $R_{E C O}^{\mathrm{ISCO}} \equiv 3 M_{\mathrm{tot}} / C$, where $C \equiv M / R$ is the compactness of the $\mathrm{ECO}$, assumed to be the same for the two merging bodies. This ansatz is corroborated by numerical analyses of NS orbits [29].

Consequently, we may define the typical frequency $f_{E C O}^{\mathrm{ISCO}}$ in the merging of two ECOs, using eq. (2) for $l=3 M_{\text {tot }} / C$ :

$$
f_{E C O}^{\mathrm{ISCO}}=\frac{C^{3 / 2}}{3^{3 / 2} \pi M_{\mathrm{tot}}} \quad(\text { for ECO })
$$




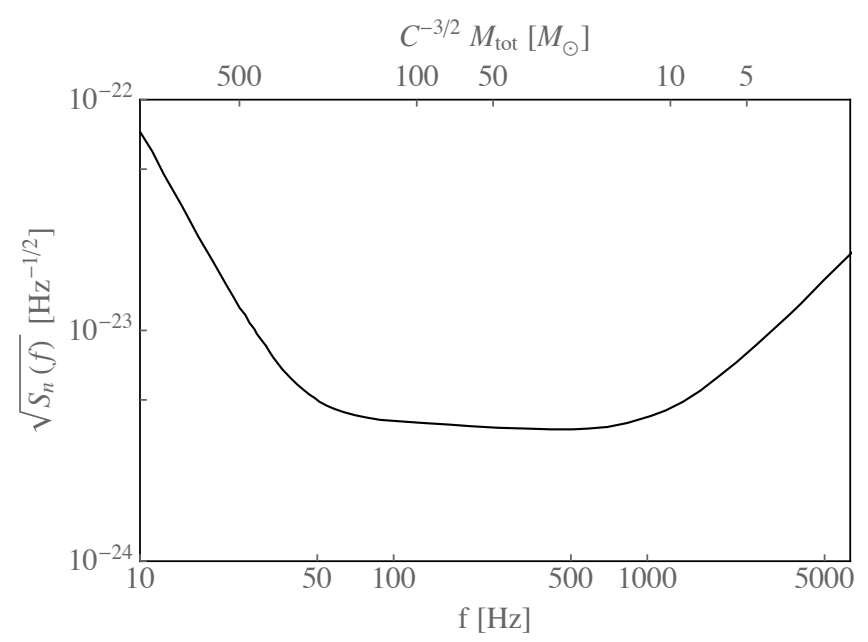

Figure 1. LIGO noise power spectral density. For a given characteristic $G W$ frequency for a binary merger, on the upper axes we show the corresponding combination of ECO compactness $C=M / R$ and total mass $M_{\mathrm{tot}}$.

Note that eq. (5) becomes equal to eq. (3), when we approach the Schwarzschild BH compactness $C_{B H}=1 / 2$.

To observe the merger and ringdown phase it is crucial that the characteristic frequency $f^{\mathrm{ISCO}}$ falls within the LIGO sensitivity range. LIGO sensitivity can be described in terms of the so-called noise power spectral density [30]. We plot this function in fig. 1. The most sensitive interval, where the signal-to-noise is maximised, is $f=[50-1000] \mathrm{Hz}$. On the upper axes of fig. 1 we also show the sensitivity in terms of $C$ and $M_{\text {tot }}$ by employing eq. (5). Thus we see that the signal-to-noise ratio is maximised for objects in the range

$$
\left(\frac{C}{0.2}\right)^{3 / 2} 1.1 M_{\odot} \lesssim M_{\text {tot }} \lesssim\left(\frac{C}{0.5}\right)^{3 / 2} 88 M_{\odot} .
$$

For a GW signal with strain $h(t)$ the expectation value of the signal-to-noise ratio is

$$
\rho^{2}=4 \int_{0}^{\infty} \frac{|\tilde{h}(f)|^{2}}{S_{n}(f)} d f,
$$

where $\tilde{h}(f)$ is the Fourier transform of the signal. As we are interested in the region of best sensitivity, in eq. (7) we have taken the case of optimal orientation (face-on, overhead source). Averaging over sky position, inclination, and polarisation, one finds that $\left\langle\rho^{2}\right\rangle$ is equal to $4 / 25$ times the expression in eq. (7). A criterion for detectability is the requirement $\rho \geqslant 8$ [31]. Assuming that most of the signalto-noise ratio is accumulated during the inspiral phase, we can use the quadrupole approximation truncated at the Newtonian order [32]

$$
\tilde{h}(f) \approx \frac{\sqrt{5 / 24}}{\pi^{2 / 3} D_{L}} M_{c}^{5 / 6} f^{-7 / 6},
$$

where

$$
M_{c}=\frac{\left(M_{1} M_{2}\right)^{3 / 5}}{\left(M_{1}+M_{2}\right)^{1 / 5}},
$$




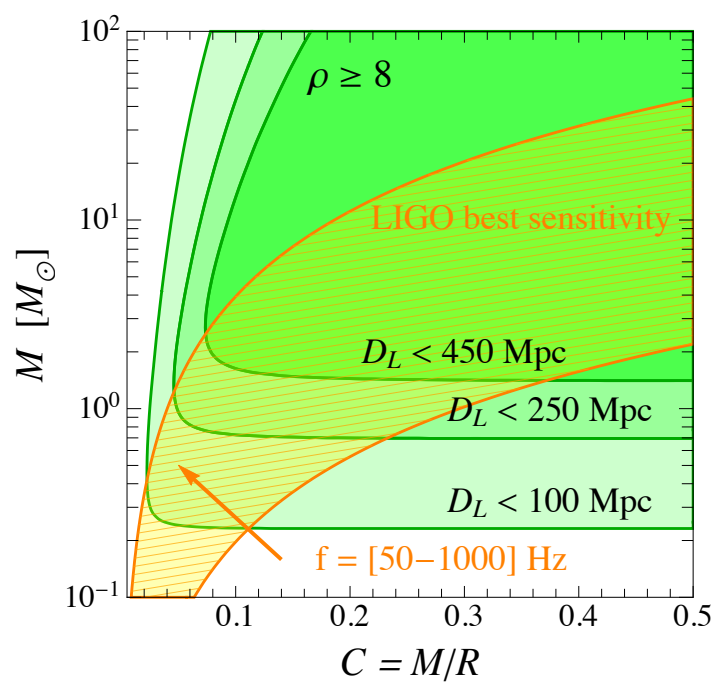

Figure 2. The LIGO best sensitivity range in ECO mass $M$ and compactness $C$, for equal mass binary $G W$ events. The yellow band corresponds to the GW frequency range $f=[50-1000] \mathrm{Hz}$, the green regions to a signal-tonoise ratio for an inspiral event occurring within the luminosity distance $D_{L}$, taking $\rho \geqslant 8$ as a criterion for detection.

is the so-called chirp mass of the system. It is important to remark that GW detectors do not measure the source-frame chirp mass, but the redshifted mass $\widetilde{M}_{c}$, related to the source-frame mass by the relation $\widetilde{M}_{c}=(1+z) M_{c}$, where $z$ is the redshift of the merging system with respect to the detector. The amplitude of a GW is proportional to the inverse of the luminosity distance $D_{L}=(1+z) D_{c}$, where the comoving distance is

$$
D_{c}=\frac{c}{H_{0}} \int_{0}^{z} \frac{d t}{E(t)}, \quad E(z)=\sqrt{\Omega_{M}(1+z)^{3}+\Omega_{k}(1+z)^{2}+\Omega_{\Lambda}},
$$

with $t_{H} \equiv 1 / H_{0}=13.969 \mathrm{Gyr}$ the Hubble time. For a given luminosity distance, and assuming standard cosmology (that corresponds to $\Omega_{k}=0, \Omega_{\Lambda}=0.7$, and $\Omega_{M}=0.3$ ), it is possible to extract the redshift $z$, and convert the detector-frame chirp mass into the source frame chirp mass.

We can use the signal-to-noise ratio criterion $\rho \geqslant 8$ to estimate the sensitivity range of the LIGO detector in terms of mass and compactness. For illustration, we take the limit of equal masses $M_{1}=$ $M_{2}=M$. Since we are limiting the analysis to the inspiral phase, eq. (8) is valid in the frequency range $f<f_{\text {ISCO }}$ and we cutoff the integral in eq. (7) at $f=f_{\text {ISCO }}$. We show our result in fig. 2. The yellow band shows the LIGO frequency sensitivity range, given in eq. (6). The green regions show the signal intensity sensitivity range (corresponding to $\rho \geqslant 8$ ), for a single GW event occurring within a distance $D_{L}$. We choose three particular values of the maximum luminosity distance, namely $D_{L}=100,250,450 \mathrm{Mpc}$. Note that the corresponding redshift is very small, ranging from $z=0.023$ for $D_{L}=100 \mathrm{Mpc}$ to $z=0.1$ for $D_{L}=450 \mathrm{Mpc}$. We therefore neglect the redshift effects in fig. 2 . The overlap between the yellow and green regions identifies the LIGO best sensitivity in ECO mass $M$ and compactness $C$. 


\subsection{Features in a Binary Mass Census}

In sect. 2.1 we focused on ECO signatures that can be extracted from the waveform of a single GW event. Here we discuss the physics information that can be extracted from statistical distributions of GW events, which will be available once a significant number of mergers have been observed.

We also remark that, even if ECO signatures were identified in single waveforms, distributions of events will be helpful to understand the ECO mass function and possibly gain knowledge about their formation. To explore this possibility we must first understand the expected distribution of known compact objects, as this will form the known 'background' distribution.

\subsubsection{On the mass distribution of compact merging binaries in the local Universe}

The mass distribution of compact object binaries can be effectively organised in a plane of the chirp mass $M_{c}$ and the symmetric mass ratio $\eta$. While the chirp mass is defined in eq. (9), the symmetric mass ratio is related to the primary and secondary binary constituent masses $M_{1}, M_{2}$ and it corresponds to the ratio between reduced and total mass.

When the population of mergers is projected onto the $M_{c}-\eta$ plane, binaries involving neutron stars and black holes (in the combinations NS-NS, NS-BH, and $\mathrm{BH}-\mathrm{BH}$ ) generally populate different regions. The mass distribution of these 'conventional' compact object binaries depends on the assumed model of stellar binary evolution. In turn, models of stellar binary evolution depend on a number of parameters that are largely unconstrained or poorly known. In the following we briefly mention some of the most relevant sources of uncertainty and features of these models, while we refer the reader to [27] for a more detailed discussion and for the relevant references.

Details of the 'common envelope (CE) phase'. The formation of a compact binary object is preceded by an evolutionary phase in which the binary system evolves enclosed into a gas envelope originated from the interactions between the donor and the companion star. The most important physical quantity describing the CE phase is the binding energy of the envelope, which in turn depends on the characteristic mass and radius of the binary system. If the binding energy is too high the stars will merge during the $\mathrm{CE}$ phase thus preventing the formation of a compact binary object. If the binding energy is too low the decrease in orbital separation during the CE phase is too slow, and the resulting compact binary object will never merge within an Hubble time. Furthermore, the formation of a CE strongly depends on the properties of the donor star. This is particularly true if the donor star is situated in the Hertzsprung gap (HG) of the Hertzsprung-Russell diagram; in this case, two alternative choices are commonly adopted: either HG stars are CE donors or they are not.

Unknown maximum mass of NS and minimal mass of BH. All observed NS have masses below 2 $M_{\odot}$, and theoretical modelling suggests that this is likely to be an upper bound. On the other hand, stellar BH, which are expected to be generated by the gravitational collapse of massive stars, have a mass distribution known to start from about $5 M_{\odot}$ and to extend to tens of solar masses. In other words, observations (mostly based on X-ray measurements) favour the existence of a mass gap between the distribution of NS and stellar BH, although it cannot be excluded that this is only the result of some observational bias.

The presence of this mass gap may seem surprising, given that the progenitor stellar mass distribution is known to be smooth. Hence, the distribution of the ensuing compact remnants might be expected to be smooth as well. However, it was shown that the presence of this mass gap can be linked to the evolutionary mechanism of dying stars. For sufficiently fast growth of the instabilities causing the stellar death, the evolution leads to two distinct outcomes: either a violent explosion that ejects most of the mass from the star, leaving a NS remnant; or a failed supernova, in which all the 


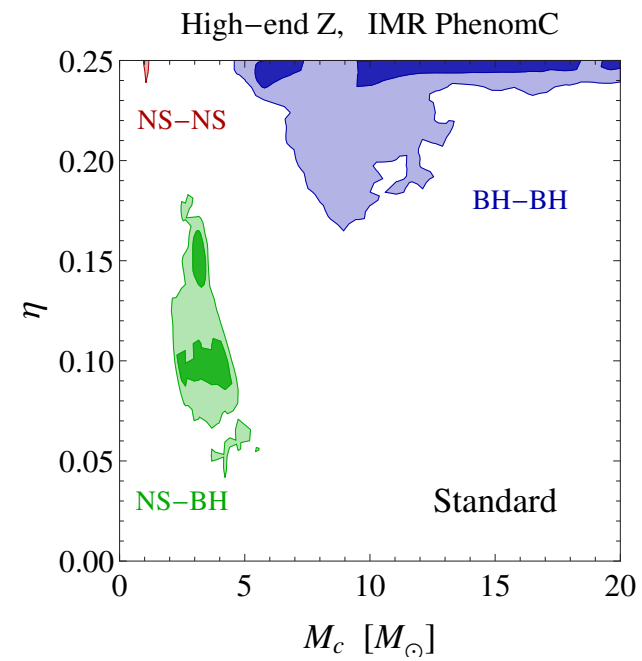

High-end Z, IMR PhenomC

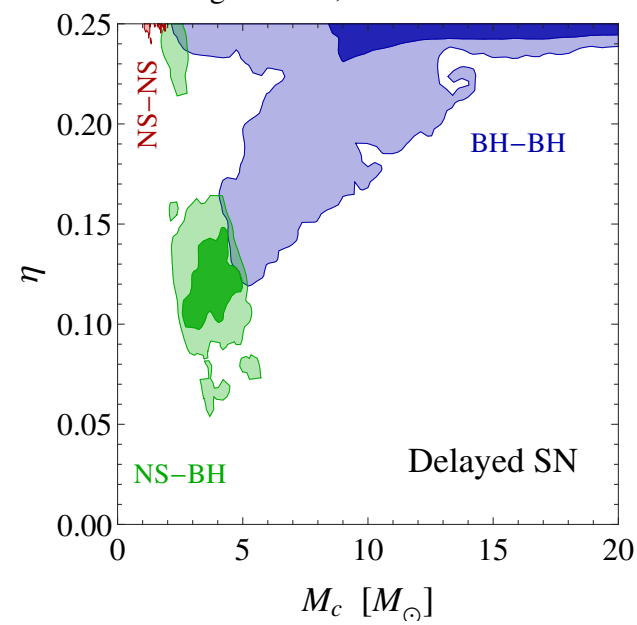

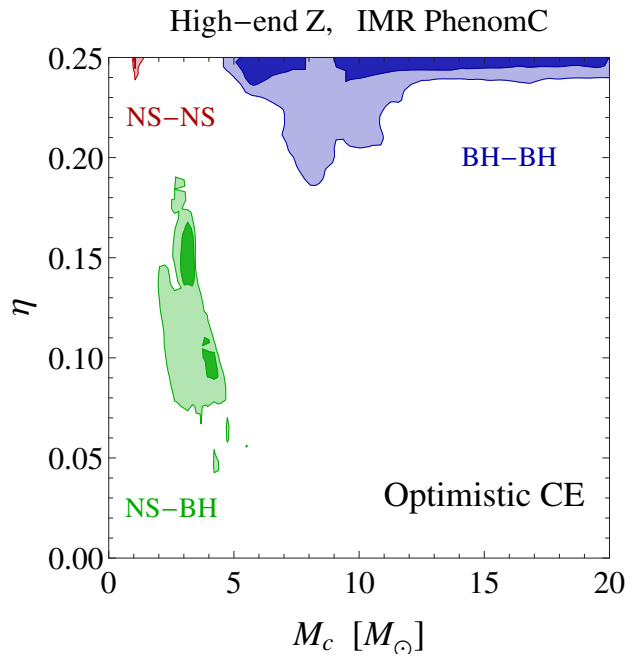

High-end Z, IMR PhenomC

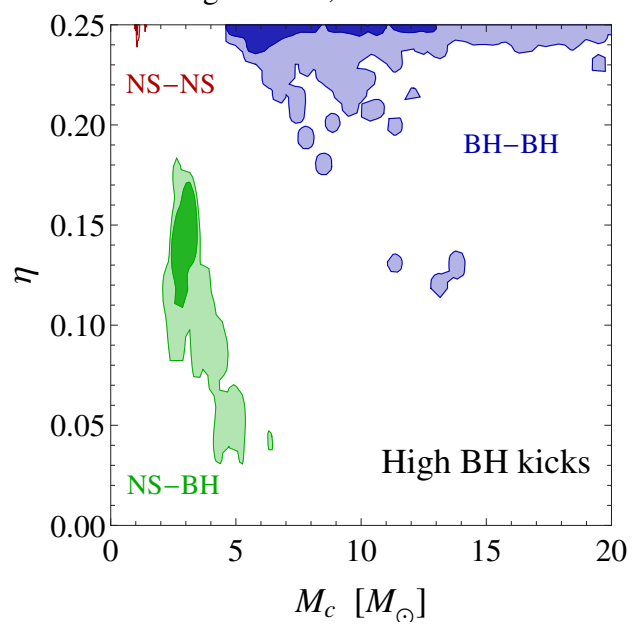

Figure 3. Binary mass distribution - in the plane chirp mass vs. symmetric mass ratio $\left(M_{c}, \eta\right)$ - for four stellar evolution models. For each distribution the contours of different colour shading represent the $68 \%$ and $99 \% \mathrm{CL}$ regions.

stellar mass collapses in a BH. A mass gap is dynamically explained, as long as the growth timescale is sufficiently short (less than about $200 \mathrm{~ms}$ ).

Physics of the explosions that form compact objects. The physics describing star evolution, supernova explosions and the subsequent formation of compact objects (either NS or BH) is plagued by many uncertainties. i) The evolution of a main sequence star toward its eventual supernova stage is affected by mass loss via stellar winds. ii) Supernova explosions may be modelled by means of different mechanism, depending on the type of instability that eventually lead to the ejection of in-falling matter. iii) Finally, the formation of a NS or BH from supernova explosions may be accompanied by a natal kick. 
Metallicity Z. The information about the metallicity - which in general describes the fraction of mass that is not in Hydrogen or Helium - is of fundamental importance to model the chemical evolution of the local Universe.

The impact of these uncertainties on the expected distribution of binary merger primaries may be estimated using simulations. The resulting distributions in the $M_{c}-\eta$ plane for these models are shown in fig. 3. Clearly, variations in the underlying model do lead to differences in the expected distributions. However, a qualitative feature that persists across all of these scenarios is that NS-NS, $\mathrm{NS}-\mathrm{BH}$, and $\mathrm{BH}-\mathrm{BH}$ mergers populate reasonably distinct regions. It is then tempting to conclude that, if future GW observations fell outside the regions in fig. 3 covered by 'conventional' mergers, we could infer evidence for an exotic population of compact objects. However, to assess this possibility it is necessary to consider more carefully the GW observables.

A GW observatory will observe the GW emitted in a binary merger. Physical properties of the binary system are then extracted by comparing the observed waveform with waveforms predicted within general relativity. Thus $M_{c}$ and $\eta$ are not themselves observables, but they may be determined from this comparison. $M_{c}$ is relatively universal and may be extracted from the inspiral phase of the wave. However, an independent extraction of $\eta$ requires consideration of the merger phase and is thus only accessible by comparison with waveforms generated numerically. If a detected waveform is studied by comparing with expected NS-NS, NS-BH, and BH-BH waveforms, then only best-fit parameters populating these regions in the $M_{c}-\eta$ plane will be found. If the waveform turns out sufficiently exotic that none of these waveforms give a good fit, then discovery would come from single-event analysis. Thus, it seems unlikely that evidence for ECOs would first arise by analyses of distributions in the $M_{c}-\eta$ plane.

However, the chirp mass may be extracted from the inspiral phase and thus does not require a detailed comparison between the observed wave and the numerical GR waveforms generated for specific scenarios. Thus, once many merger events have been observed, it may be possible to search for a new distribution of ECOs by considering the chirp mass alone, without relying too heavily on specific expected waveforms. In fig. 4 we show the four distributions of fig. 3 projected onto the chirp mass axes alone. To make a concrete connection with observations, we superimpose the expected LIGO sensitivity as a function of the chirp mass. At each value of red-shifted chirp mass - under the simplifying assumption $M_{1}=M_{2}$ - we computed the maximum luminosity distance at which the signal-to-noise ratio in eq. (7) reaches the detectability threshold $\rho=8$. The corresponding redshift, evaluated according to eq. (10), allows us to reconstruct the maximum volume $V$ inside which the analysed binary could be seen. The expected upper limit on the merger rate density, after an operating time $T$, is $\mathcal{R}=1 /(\epsilon V T)$, where $\epsilon$ is an experimental efficiency which we take to be $30 \%$.

Note that this result, although strictly valid only for the case of equal-mass BH-BH mergers, should give a correct order-of-magnitude estimate of the expected LIGO sensitivity.

Clearly, there are large variations in the expected distributions based on the underlying model. However, in three of the four choices an identifiable chirp mass gap appears between the NS-NS and $\mathrm{BH}-\mathrm{BH}$ distributions. This mass gap does not arise in the model with delayed SN explosion, as also clear from the corresponding mass distribution in fig. 3. However, when projected onto the chirp mass axes, the merger rate density of $\mathrm{BH}-\mathrm{BH}$ binaries populating the mass gap turns out to be extremely low, order of magnitudes below the LIGO sensitivity. Finally, we note that the BH-BH binary merger rate expected from the scenario with high natal $\mathrm{BH}$ kicks is smaller than for the other models and appears to be in some tension with the observation of GW150914, given the LIGO sensitivity. Thus, the high natal BH kick scenario already appears disfavoured. These simulations thus seem to suggest that a mass gap is likely for binary mergers of known objects. 


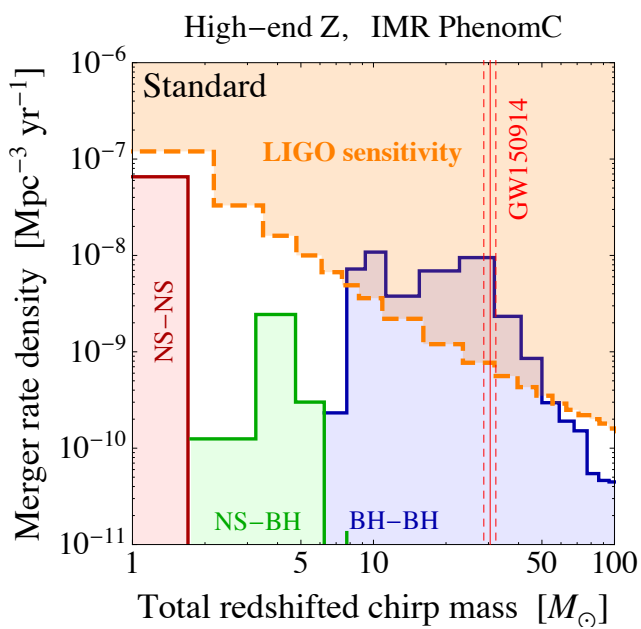

High-end Z, IMR PhenomC

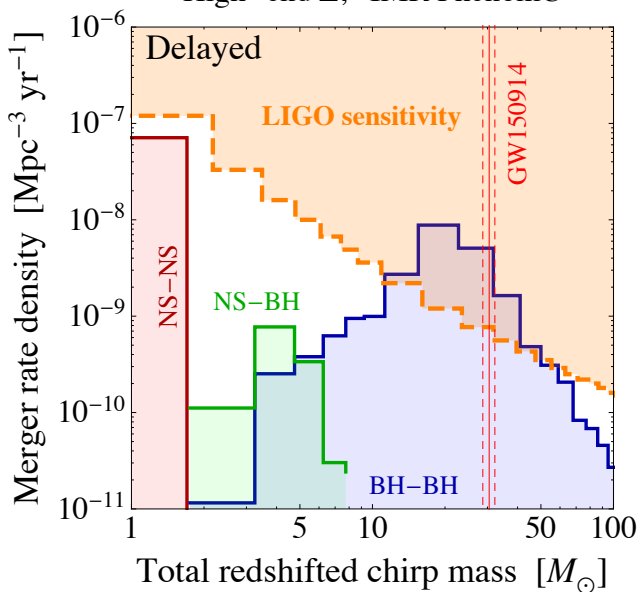

High-end Z, IMR PhenomC

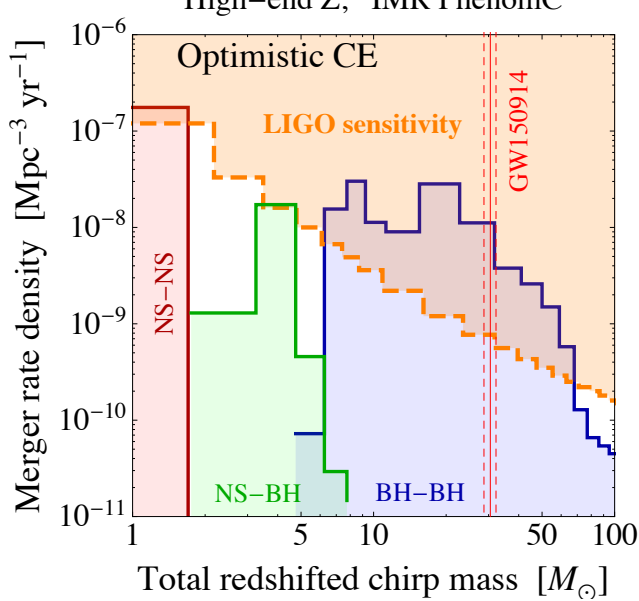

High-end Z, IMR PhenomC

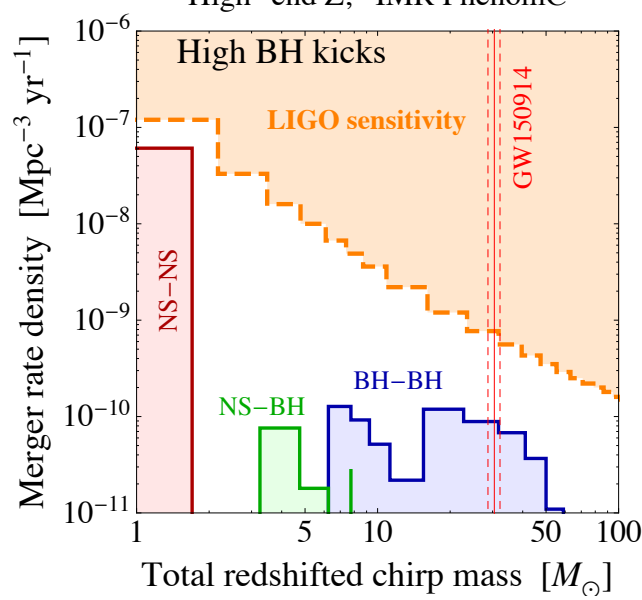

Figure 4. Merger rate density as a function of the redshifted chirp mass for four stellar evolution models. In each case, we show the distributions for NS-NS, NS-BH and BH-BH event rates. The vertical red line corresponds to the event GW150914, with measured detector-frame chirp mass $M_{c}=30.5_{-1.8}^{+1.7} M_{\odot}$ and source redshift $z=$ $0.093_{-0.036}^{+0.028}$. Also shown is the LIGO detection sensitivity, obtained with the estimate described in the text.

There is no reason to expect ECOs to have a mass distribution that exhibits a mass gap in precisely the same location. Thus, events filling the gap in the chirp mass distribution would point towards a population of ECOs. This is especially true if the waveforms appear to be of the NS-NS or NS-BH type, because massive ECOs with compactness $C<1 / 2$ could resemble NS, although with anomalously large masses.

To make a quantifiable assessment of this possibility would require not only a better understanding of the systematic uncertainties in the expected distribution of known objects, but also concrete predictions for the distribution of ECOs. As this distribution will depend in detail on the formation mechanism it is highly model dependent and would require treatment on a case-by-case basis for each particle physics model. As this is beyond the scope of this work we will simply speculate that the 
comparison of the observed distribution of chirp masses with that expected from theoretical models can give some information about a possible population of ECOs. Unfortunately, the large astrophysical uncertainties in the modelling of the 'background' are a severe limiting factor for the discovery of a 'signal'.

\section{Test of the Area Theorem}

Hawking's area theorem [34] states that the sum of the horizon areas of a system of BHs never decreases. This remarkable result follows directly from general relativity and the null energy condition. However, once we use the thermodynamical identification of a BH temperature (inversely proportional to mass, $T=1 / 8 \pi M[35,36]$ ) and BH entropy (proportional to area, $S=A / 4$ [37-39]), Hawking's theorem acquires a new interpretation [40-42]. The statement about the ever increasing area can be viewed as a generalised second law of thermodynamics for the ever increasing entropy.

This thermodynamical interpretation of the area theorem has a deep physical significance that goes beyond classical general relativity. Indeed, the area theorem is apparently violated by the quantum process of Hawking's radiation. During BH evaporation, conservation of energy implies that the $\mathrm{BH}$ mass must decrease, hence the $\mathrm{BH}$ area must decrease as well. This contradiction with the area theorem is resolved in the context of the generalised second law of thermodynamics. Once we account for the entropy of the emitted matter and radiation, and not only the entropy associated with the $\mathrm{BH}$ area, the second law of thermodynamics is respected by $\mathrm{BH}$ evaporation. All this to say that any observational test of the area theorem is a way of probing the fundamental principles of general relativity and statistical mechanics.

It is remarkable that GW detection can indeed provide a test of the area theorem. The horizon area of a Kerr $\mathrm{BH}$ of mass $M$ and angular momentum $J$ is given by

$$
A=8 \pi M^{2}\left(1+\sqrt{1-a^{2}}\right) \quad a \equiv \frac{J}{M^{2}} .
$$

For the process of two $\mathrm{BH}$ with horizon areas $A_{1,2}$ merging into a final $\mathrm{BH}$ of area $A_{f}$, Hawking's theorem predicts

$$
A_{f}>A_{1}+A_{2} \quad \text { (area theorem) }
$$

The area theorem does not only provide a test of fundamental principles, but can also probe the existence of ECOs. In order to understand how ECOs could give an apparent violation of the area theorem, if erroneously taken for BHs, it is useful to clarify the physical content of the area theorem when applied to merger events. eq. (12) can be written as a lower bound on the final BH mass

$$
M_{f}>\sqrt{M_{1}^{2} s_{1}+M_{2}^{2} s_{2}}, \quad s_{1,2} \equiv \frac{1+\sqrt{1-a_{1,2}^{2}}}{1+\sqrt{1-a_{f}^{2}}} .
$$

The absence of naked singularities (aka cosmic censorship conjecture [43]) requires $0 \leq a \leq 1$, which implies $s_{1,2}>1 / 2$. Using this condition in eq. (13), we find

$$
M_{f}>\sqrt{\frac{M_{1}^{2}+M_{2}^{2}}{2}} .
$$

In words, Hawking's theorem implies a simple lower bound on the mass $M_{f}$ of the BH resulting from the merging of two BHs with masses $M_{1,2}$. 
Another way of reading eq. (12) is as an inequality on the efficiency in GW emission, $\epsilon \equiv\left(M_{1}+\right.$ $\left.M_{2}-M_{f}\right) /\left(M_{1}+M_{2}\right)$

$$
\epsilon<1-\frac{\sqrt{s_{1}+r^{2} s_{2}}}{1+r}, \quad r \equiv \frac{M_{2}}{M_{1}}
$$

The upper bound on $\epsilon$ is maximised for maximally-rotating initial BHs and non-rotating final BH $\left(s_{1,2}=1 / 2\right)$, giving $\epsilon<1-\sqrt{\left(1+r^{2}\right) / 2} /(1+r)$. In turn, this is optimised for $r=1$, giving $\epsilon<1 / 2$. In the special case of slowly-rotating initial BHs $\left(1 \leq s_{1}=s_{2} \leq 2\right)$, the maximum upper bound is $\epsilon<1-\sqrt{1+r^{2}} /(1+r)$, which is optimised for $r=1$ giving $\epsilon<1-1 / \sqrt{2}$.

To summarise, the area theorem prescribes a maximum efficiency in $G W$ emission for the merging process of two BHs. An apparent violation of the theorem could occur if the merging objects are not really BHs and emit other forms of energy, which goes undetected and exceeds the bound in eq. (15). In other words, the apparent violation of the area theorem would occur in the same way as for Hawking's BH evaporation. Emission of non-gravitational radiation leads to entropy production, left unaccounted for by the area theorem. As a result, the area theorem is not satisfied, although the generalised second law of thermodynamics holds valid.

As a concrete example of observational test of the area theorem, let us consider the detection of GW150914, for which the extracted parameters are $M_{1}=36_{-4}^{+5} M_{\odot}$ and $a_{1}=0.3_{-0.3}^{+0.5}$ for the primary, $M_{2}=29 \pm 4 M_{\odot}$ and $a_{2}=0.5_{-0.4}^{+0.5}$ for the secondary, and $M_{f}=62 \pm 4 M_{\odot}$ and $a_{f}=0.67_{-0.07}^{+0.05}$ for the final $\mathrm{BH}$ [33]. This gives

$$
A_{1}+A_{2}=(2.2 \pm 0.2) \times 10^{5} \mathrm{~km}^{2}, \quad A_{f}=(3.7 \pm 0.4) \times 10^{5} \mathrm{~km}^{2}
$$

where we have taken into account the error correlation among the input parameters using the information in [33]. The result in eq. (16) shows that the observation of GW150914 has verified eq. (12), hence Hawking's area theorem, well within errors. This is also visualised in fig. 5, where we show how the LIGO result falls within the region of plane $A_{1}+A_{2}$ vs $A_{f}$ allowed by the area theorem. In the same plane we also show the region excluded by the condition on conservation of energy $M_{f}<M_{1}+M_{2}$, which corresponds to $A_{f}<\left(\sqrt{A_{1} / s_{1}}+\sqrt{A_{2} / s_{2}}\right)^{2}$. Recalling that $s_{1,2}>1 / 2$, we obtain

$$
A_{f}<4\left(A_{1}+A_{2}\right) \quad \text { (energy conservation). }
$$

It should be stressed that the experimental determination of $A_{f}$ in eq. (16) is not independent of $A_{1}+A_{2}$. Once one assumes that the merging binaries are $\mathrm{BH}$ and fixes the initial parameters $\left(M_{1,2}\right.$, $a_{1,2}$, the relative angles between the spin vectors, the angular velocity, and orbit eccentricity), the values of $M_{f}$ and $a_{f}$ are uniquely determined by the general relativistic calculation. A genuine test of the area theorem would require an independent extraction of the initial and final BH parameters. This is not impossible as the inspiral and ringdown phases are mostly sensitive to initial and final $\mathrm{BH}$ parameters, respectively.

Future GW observations of BH mergers will further test the area theorem. What if these observations do not agree with the theorem's expectations? Unless one is ready to abandon the basic principles of general relativity, measurements of apparent violations of the area theorem can be used to argue for the existence of ECOs. Depending on their nature, ECOs could emit significant fractions of their energy in non-gravitational forms, in practice exceeding the bound in eq. (15). This can happen if they emit intense electromagnetic radiation in collimated narrow beams not pointing towards the line of sight, or if they radiate particles (such as dark photons or light axion-like particles) that remain invisible to us.

The possibility of testing the area theorem has a theoretical interest independent of ECOs, since its validity is not guaranteed in various extension of general relativity (GR). The key observation for 


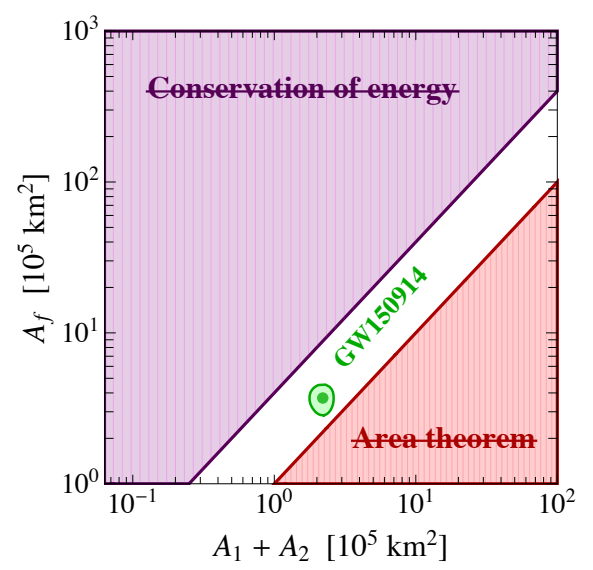

Figure 5. In the plane of the horizon areas of initial $\left(A_{1}+A_{2}\right)$ and final $\left(A_{f}\right)$ BH binary mergers, the dashed regions indicate where Hawking's area theorem is violated $\left[A_{f}<A_{1}+A_{2}\right]$ and where energy conservation does not hold $\left[A_{f}>4\left(A_{1}+A_{2}\right)\right]$. The result from GW150914 corresponds to the green area $($ at $90 \% C L)$ and lies within the allowed region (white band). The correlation between the extraction of $A_{f}$ and $A_{1}+A_{2}$ is not taken into account.

proving the theorem is that in GR the null energy condition - that is $T_{a b} k^{a} k^{b} \geqslant 0$ for any null vectors $k^{a}$ - implies, as a direct consequence of Einstein equations $\left(R_{a b}-g_{a b} R / 2=8 \pi G_{N} T_{a b}\right)$, the null convergence condition $R_{a b} k^{a} k^{b} \geqslant 0$. In turn, the null convergence condition ensures, by means of the Raychaudhuri equation describing geodesics congruence, the validity of the area theorem. The proof is not valid in extended gravity theories, when the field equations differ from those of GR. This is the case of the Brans-Dicke theory (and, more generally, scalar-tensor gravity) [44, 45], in which Einstein equations are modified by new terms beyond the energy-momentum tensor involving the scalar field.

As a consequence, the null energy condition does not imply anymore the null convergence condition, thus invalidating Hawking's argument. Even if static asymptotic BH must recover GR solutions (the no-hair theorem), during the dynamical stages of the merging process the validity of the area theorem is not guaranteed. In terms of energy balance, this corresponds to the emission - in addition to tensor GW perturbations - of the extra scalar degrees of freedom.

In conclusion, the search for violations of the area theorem can be used as a tool to confirm potential ECO discoveries and to derive information on undetected forms of radiation. Furthermore, testing the area theorem is an interesting task independently of searches for ECOs, as it offers a probe of fundamental principles.

\section{References}

[1] B. P. Abbott et al. [LIGO Scientific and Virgo Collaborations], Phys. Rev. Lett. 116 (2016) 6, 061102.

[2] E. O. Kahya and S. Desai, arXiv:1602.04779 [gr-qc].

[3] X. F. Wu et al., arXiv:1602.01566 [astro-ph.HE].

[4] N. Yunes, K. Yagi and F. Pretorius, arXiv:1603.08955 [gr-qc].

[5] M. Liu, Z. Zhao, X. You, J. Lu and L. Xu, arXiv:1604.06668 [gr-qc].

[6] R. Konoplya and A. Zhidenko, arXiv:1602.04738 [gr-qc]. 
[7] J. W. Moffat, arXiv:1603.05225 [gr-qc].

[8] J. Vainio and I. Vilja, arXiv:1603.09551 [astro-ph.CO].

[9] V. Cardoso, E. Franzin and P. Pani, Phys. Rev. Lett. 116, 171101 (2016).

[10] S. B. Giddings, arXiv:1602.03622 [gr-qc].

[11] M. Sasaki, T. Suyama, T. Tanaka and S. Yokoyama, arXiv:1603.08338 [astro-ph.CO].

[12] S. Bird, I. Cholis, J. B. Muñoz, Y. Ali-Haïmoud, M. Kamionkowski, E. D. Kovetz, A. Raccanelli and A. G. Riess, arXiv:1603.00464 [astro-ph.CO].

[13] S. Clesse and J. García-Bellido, arXiv:1603.05234 [astro-ph.CO].

[14] T. E. Collett and D. Bacon, arXiv:1602.05882 [astro-ph.HE].

[15] P. Bicudo, arXiv:1602.04337 [gr-qc].

[16] D. Blas, M. M. Ivanov, I. Sawicki and S. Sibiryakov, arXiv:1602.04188 [gr-qc].

[17] E. Calabrese, N. Battaglia and D. N. Spergel, arXiv:1602.03883 [gr-qc].

[18] J. García-Bellido, S. Nesseris and M. Trashorras, arXiv:1603.05616 [astro-ph.CO].

[19] M. Schreck, arXiv:1603.07452 [gr-qc].

[20] M. Arzano and G. Calcagni, arXiv:1604.00541 [gr-qc].

[21] V. Branchina and M. De Domenico, arXiv:1604.08530 [gr-qc].

[22] P. S. B. Dev and A. Mazumdar, arXiv:1602.04203 [hep-ph].

[23] J. Jaeckel, V. V. Khoze and M. Spannowsky, arXiv:1602.03901 [hep-ph].

[24] K. Langaeble, A. Meroni and F. Sannino, arXiv:1603.00230 [hep-ph].

[25] L. Lehner, S. L. Liebling, C. Palenzuela, O. L. Caballero, E. O'Connor, M. Anderson and D. Neilsen, arXiv:1603.00501 [gr-qc].

[26] A. Arvanitaki, M. Baryakhtar, S. Dimopoulos, S. Dubovsky and R. Lasenby, arXiv:1604.03958 [hep-ph].

[27] G. F. Giudice, M. McCullough and A. Urbano, JCAP 1610 (2016) no.10, 001.

[28] P. Ajith et al., Phys. Rev. Lett. 106 (2011) 241101.

[29] D. Lai and A. G. Wiseman, Phys. Rev. D 54 (1996) 3958.

[30] P. Ajith, Phys. Rev. D 84, 084037 (2011).

[31] M. Dominik et al., Astrophys. J. 806, no. 2, 263 (2015).

[32] S. Khan, S. Husa, M. Hannam, F. Ohme, M. Pürrer, X. Jimènez Forteza and A. Bohè, Phys. Rev.

D 93, no. 4, 044007 (2016).

[33] B. P. Abbott et al. [LIGO Scientific and Virgo Collaborations], arXiv:1602.03840 [gr-qc].

[34] S. W. Hawking, Phys. Rev. Lett. 26 (1971) 1344.

[35] S. W. Hawking, Nature 248 (1974) 30.

[36] S. W. Hawking, Commun. Math. Phys. 43 (1975) 199 [Commun. Math. Phys. 46 (1976) 206].

[37] J. D. Bekenstein, Lett. Nuovo Cim. 4 (1972) 737.

[38] J. D. Bekenstein, Phys. Rev. D 7 (1973) 2333.

[39] J. D. Bekenstein, Phys. Rev. D 9 (1974) 3292.

[40] T. Jacobson, G. Kang and R. C. Myers, Phys. Rev. D 49 (1994) 6587.

[41] J. D. Bekenstein, gr-qc/9409015.

[42] T. Jacobson, G. Kang and R. C. Myers, Phys. Rev. D 52 (1995) 3518.

[43] R. Penrose, Riv. Nuovo Cim. 1 (1969) 252 [Gen. Rel. Grav. 34 (2002) 1141].

[44] G. Kang, Phys. Rev. D 54, 7483 (1996).

[45] V. Faraoni, Entropy 12, 1246 (2010). 